Revue d'histoire de l'Amérique française

REVUE D.HISTOIRE DE L'AMÉRIQUE FRANÇAISE

\title{
Le Globe and Mail et le Québec, 1935-1939
}

\section{Brian J. Young}

Volume 25, numéro 1, juin 1971

URI : https://id.erudit.org/iderudit/303045ar

DOI : https://doi.org/10.7202/303045ar

Aller au sommaire du numéro

Éditeur(s)

Institut d'histoire de l'Amérique française

ISSN

0035-2357 (imprimé)

1492-1383 (numérique)

Découvrir la revue

\section{Citer cette note}

Young, B. J. (1971). Le Globe and Mail et le Québec, 1935-1939. Revue d'histoire de l'Amérique française, 25(1), 96-105. https://doi.org/10.7202/303045ar d'utilisation que vous pouvez consulter en ligne.

https://apropos.erudit.org/fr/usagers/politique-dutilisation/ 


\title{
LE GLOBE AND MAIL ET LE QUÉBEC, 1935-1939
}

\author{
Brian J. Young \\ University of Vermont
}

Les années 1930 furent une période de déboires pour le Canada. Les gouvernements de R. B. Bennett et de Mackenzie King éprouvèrent toutes sortes de difficultés à lutter contre le chômage, à répandre le bien-être social, les mesures de secours et à atténuer les tensions sociales nées des difficultés du temps. Les tiers partis, le C.C.F., le Crédit social et le Reconstruction Party, ont tourné cette situation à leur profit et ont obtenu plus de $20 \%$ des votes au cours des élections fédérales de 1935 . De nouveaux leaders provinciaux ont menacé les structures du fédéralisme. La Colombie britannique, l'Alberta, l'Ontario et le Québec se donnèrent des chefs aux allures démagogiques qui exigèrent des concessions de la part du gouvernement central.

Ce ne fut pas seulement aux milieux dépossédés de la société canadienne que la dépression posa un défi ; les capitalistes regardaient avec crainte les nouveaux gouvernements provinciaux de Patullo, Aberhart, Hepburn et Duplessis qui se faisaient les avocats de l'autonomie des provinces et brandissaient la menace de l'intervention de l'Etat. Le régionalisme, le radicalisme, ajoutés au malaise général, avaient remplacé la confiance des bâtisseurs de nations, confiance qui avait prévalu pendant la décennie précédente. Ce climat de doute et d'incertitude poussa à l'action quelques capitalistes. George McCullagh fut un de ceux-là. 
Né en 1905 à London, en Ontario, d'un milieu ouvrier, McCullagh personnifiait la légende de Horatio Alger. Après six mois seulement d'école secondaire, McCullagh passa rapidement de la Merchants Bank au service de diffusion du journal Globe; dès 1928, il était assistant du directeur financier. Il quitte le Globe cette même année pour se consacrer entièrement à la bourse. A l'âge de 30 ans, devenu millionnaire, il était prêt pour de nouvelles entreprises. Il réussit à gagner la confiance du solitaire William Wright qui s'était amassé une fortune colossale dans l'exploitation des mines d'or Lakeshore et WrightHergreaves et à le convaincre d'acquérir les deux quotidiens du matin de Toronto. Wright acheta le Globe pour $\$ 1,300,000$ et le Mail and Empire pour $\$ 2,225,000$, les fusionna sous le titre de Globe and Mail en novembre 1936 et en confia la direction à McCullagh. ${ }^{1}$ En tant que directeur du journal, McCullagh veille de près aux éditoriaux. Chaque jour il réunit son comité de rédaction et fait souvent récrire les éditoriaux (quelquefois même les bulletins de nouvelles) pour les rendre conformes à son point de vue. Dès le début, McCullagh affirma que son journal adopterait une orientation politique indépendante. Généralement favorable aux libéraux en 1936 et 1937, le Globe and Mail fut indépendant en 1939 et ne soutint aucun parti aux élections de 1940. Plus tard, pendant la guerre, il donna son appui au parti conservateur.

L'une des premières tâches de McCullagh fut de faire du Globe and Mail le porte-parole des hommes d'affaires torontois, plus particulièrement des intérêts miniers de la ville. McCullagh n'était évidemment pas le seul à avoir misé sur le "boom" minier du nord de l'Ontario et du Québec dans les années 1920 et 1930. L'afflux des capitaux américains et l'orientation des échanges selon l'axe nord-sud dans le secteur des produits miniers et des pâtes et papiers avaient accru l'importance de Toronto. Le Globe and Mail allait devenir l'organe de la nouvelle élite; au cours d'un dîner offert en son honneur en 1936, McCullagh fit remarquer: "I have had some material luck associated with the mining game and I have been moved by a great desire, a burning inspiration, to really do some good, to make this country more secure for the generations to come." ${ }^{2}$ Sans relâche le Globe and Mail plaide en faveur d'une taxation modérée, de la paix dans l'industrie, de la stabilité des finances et d'un climat favorable à l'investissement. Il va sans dire que ces conditions présupposaient

\footnotetext{
1 Pierre Berton, "The Amazing Career of George McCullagh", Maclean's (15 janvier 1949), 44.

2 Globe and Mail, 3 novembre 1936.
} 
un prolétariat passif, ce qui explique pourquoi le Globe and Mail combattit farouchement la pénétration au Canada des syndicats industriels américains.

Il est intéressant d'étudier l'attitude du journal torontois et de son directeur face au Québec de la fin des années 1930. En effet, l'économie québécoise gravitait dans l'orbite de Toronto et le Globe and Mail décrivait en détail les occasions d'investissements dans les mines, les centrales électriques et les usines de pâtes et papiers de la province. D'une manière générale, $\mathrm{McCul}$ lagh se montra très satisfait de la collaboration que le gouvernement Duplessis apportait aux hommes d'affaires. Le Globe and Mail rapporte avec plaisir la déclaration du ministre des Finances de la province qui prévoyait un excédent de 3 millions de dollars pour l'année 1938. Le journal applaudit aux mesures que Duplessis avait prises pour empêcher la conversion des "bonds" du trésor de la province ou même le refus de les payer. Elles étaient de nature à améliorer la position déjà favorable dont bénéficiait la province auprès des investisseurs. Le Globe and Mail rappela à ses lecteurs que la croissance économique est liée à l'industrie minière et que les intérêts du Québec s'identifient à ceux des propriétaires de mines. Une prévision de la production minière fixée à $\$ 67,000,000$ en 1938 rassurait les investisseurs torontois en même temps que la déclaration du ministre des Mines, Onésime Gagnon, à l'effet que le gouvernement du Québec apporterait à l'industrie minière une contribution même supérieure à celle de $1937 .^{3}$

Dans ses rubriques financières sur le Québec, le Globe and Mail se tenait à l'affût de toute législation qui serait défavorable à l'industrie minière. Il en fut ainsi du "bill" 5 présenté à la session parlementaire de 1937. Ce "bill" forçait toutes les sociétés minières québécoises exploitant le domaine public à opérer sous l'empire d'une charte provinciale et inspira les plus sévères menaces au journal torontois. Dans des éditoriaux tels que "Mines a National Asset" et "Hits at National Prosperity", le Globe and Mail avertit le gouvernement Duplessis que c'était l'Ontario, grâce à l'énergie et à l'optimisme d'hommes d'expérience faisant affaires à Toronto, qui avait financé et développé l'industrie minière du Québec. ${ }^{4}$ Les mines étaient une industrie "national", étant donné que seulement 10 à $15 \%$ des 60 à 75 millions de dollars investis dans les mines du Québec provenaient de cette province. Le Globe and Mail qualifia de "raciales" ces

3 Ibid., 5 janvier 1939.

4 Ibid., 31 juillet 1937. 
mesures qui imposaient des conditions provinciales sur les investissements, et prédit que la stagnation au Québec suivrait inévitablement cette menace "national", cet exemple éclatant de régionalisme racial. On invitait le Québec à se joindre aux autres provinces dans un effort pan-canadien qui ne connaîtrait pas de frontière de foi, de religion ou de province. ${ }^{5}$ Neuf mois plus tard, quand le premier ministre, après consultation avec les dirigeants des industries minières, annonça son consentement à amender le "bill" 5, le Globe and Mail félicita le Québec d'avoir encore une fois adopté un point de vue "national".

Bien que couvrant largement les questions économiques québécoises, le Globe and Mail ne mentionnait que rarement les autres aspects de la vie des Canadiens français. N'ayant pas de correspondant permanent ni à Montréal ni à Québec, le Globe and Mail se fiait à la Gazette de Montréal pour tout ce qui concernait les nouvelles du Québec. Lorsque l'on faisait mention des Canadiens français, c'était généralement pour vanter leur modération et leur existence pastorale. Le Globe and Mail s'accordait avec John Bassett, président de la Gazette de Montréal, pour affirmer que les Français du Québec forment le peuple le plus chaleureux et le plus raisonnable au monde et que leur courtoisie et leur hospitalité sont proverbiales. ${ }^{6}$ Dans une autre allusion à la vie sociale des Canadiens français, le Globe and Mail faisait l'éloge de Louis Hébert. "Avec une simple bêche, il était parvenu à tirer du sol de quoi subvenir aux besoins de sa femme et de ses trois enfants sans aucune assistance d'un gouvernement paternaliste. La précocité du mariage, le profond attachement à la famille, les chansons et danses folkloriques, c'étaient là les traits d'une société faite de simplicité, de dévouement et de charité." 7 Cette conception du Québec venait du fait que le Globe and Mail était en faveur d'une vie rurale, ou, tout au moins, du maintien des valeurs rurales traditionnelles. Sur la ferme, les vertus d'économie, de labeur, de courage et d'honnêteté n'avaient pas été affaiblies par le confort de la civilisation. Préoccupé par les agitations dans les villes, le Globe and Mail suggéra d'établir les citadins en chômage sur des terres, dans le nord, afin qu'ils imitent cette vie. Un éditorial intitulé "Few Reds on the Farm" était fort explicite:

The agriculturist is a staunch supporter of Church and State, of law and order, and he is, first and foremost, devoted to

\footnotetext{
5 Ibid.

6 Ibid., 5 mars 1937.

7 Ibid., 29 juin 1939.
} 
his home and his family. In these circumstances there need be little fear of communism taking root in rural Canada. ${ }^{8}$

Ce caractère doux et rural qu'il accordait volontiers au peuple canadien-français, le Globe and Mail l'attribuait de même aux hommes politiques du Québec. Ernest Lapointe possédait toutes les qualités désirables d'un Canadien français. Le ministre de la Justice était compétent et courtois et d'une intelligence hors ligne. Personnage robuste aux cheveux noirs, ce bon avocat du bas Saint-Laurent devait son ascension à son travail, à son intégrité, à sa courtoisie et à son esprit chevaleresque. ${ }^{9}$ Le Globe and Mail établissait une très nette différence entre les hommes politiques représentatifs du véritable esprit canadien-français et les autres. Autour de Lapointe, dans la première catégorie, on trouvait tous les modérés. Le Globe and Mail prétendait que des déclarations faites par des hommes politiques, tel Aurélien Bélanger, reflétaient l'opinion publique canadienne-française :

Nous sommes loyaux parce que nous sommes reconnaissants.

Nous sommes loyaux parce que nous croyons que nos intérêts sont liés à ceux de la Grande-Bretagne. Nous sommes loyaux parce que nous croyons que, mieux que toute autre allégeance, le drapeau britannique garantit nos droits. ${ }^{10}$

Son discours était logique, commentait le "Globe and Mail". Il est temps de cesser les insultes à l'intelligence des Canadiens français. D'autre part, des leaders, tels Georges Héon, député d'Argenteuil, et Camillien Houde, représentaient des éléments dangereux. En refusant son appui à Arthur Meighen au congrès du parti conservateur de 1938, Héon avait présenté un défi difficile aux Anglo-Canadiens de l'est et de l'ouest du pays. Ce sont des exemples d'entêtement comme celui de Héon qui amenèrent le Globe and Mail à se demander si le Canada n'avait pas besoin d'une politique d'immigration capable de rétablir l'équilibre de l'élément britannique, équilibre presque perdu. ${ }^{11}$ Une autre tactique consistait à isoler des radicaux comme Houde en invoquant le fait qu'ils ne représentaient qu'un petit groupe de mécontents. La réélection de Houde à la mairie de Montréal en décembre 1938 scandalisa le Globe and Mail. Il expliqua cette victoire en assurant qu'une fois de plus, elle révélait les vues superficielles des hommes politiques et la médiocrité de la vie publique. ${ }^{12}$

8 Ibid., 1er janvier 1938.

9 Ibid., 20 février 1939.

10 Ibid., 25 mars 1939.

11 Ibid., 6 juillet 1938 .

12 Ibid., 14 décembre 1938. 
McCullagh n'eut jamais tout à fait confiance en Maurice Duplessis, le premier ministre. Il appréciait l'attention spéciale que Duplessis portait à la vie rurale, ainsi que son opposition vigoureuse au communisme et au radicalisme. Il approuvait aussi les rapports étroits qui existaient entre le gouvernement et les intérêts engagés dans l'exploitation des mines, des pâtes et papiers et de l'énergie hydro-électrique. L'attitude du Globe and Mail envers la politique de Duplessis montre clairement l'évolution des idées de McCullagh. Son désenchantement pour la vie publique au Canada et son admiration pour l'autoritarisme de Duplessis grandissaient parallèlement. Le journal de McCullagh vantait la manière simple et directe que Duplessis utilisait en politique, de même que son refus de se laisser entraîner en dehors de l'essentiel.

One of the virtues of Parliamentary procedure is its orderliness. Mr. Duplessis' method of breaking up the debate is not to be commended there should be order of a kind which will put essentials first...13

Le Globe and Mail avait pris une attitude ambivalente vis-àvis la "loi du cadenas". Le journal avait souvent préconisé que le gouvernement fédéral désavoue la législation bancaire de l'Alberta, étant donné que cette législation vise à détruire les relations d'affaires entre les provinces et la "national unity". Pourtant, en 1937, le Globe and Mail ne participa pas activement au débat sur la "loi du cadenas" ; il s'agissait d'un problème provincial. Bien que cette loi ait été un assaut violent contre la démocratie et une loi “non britannique", le point litigieux qu'elle soulevait devait être résolu par le seul peuple québécois. En dénonçant la pression extérieure exercée contre la "loi du cadenas", McCullagh interprétait la législation du Québec comme un bouclier contre le communisme. Cette loi empêcherait la lutte des classes de se transformer en batailles: le Globe and Mail rappelait à ses lecteurs que si les communistes l'emportaient, ils mettraient le cadenas sur les idées politiques de M. Duplessis. ${ }^{14}$ Fortement préoccupé par la poussée du radicalisme, McCullagh était convaincu que les communistes s'infiltraient parmi les immigrants, les jeunes, les chômeurs et les syndiqués. Il craignait que les effets heureux de la "loi du cadenas" qui aurait chassé les communistes hors du Québec, les déversent sur l'Ontario, tout spécialement dans les mines du nord. L'Ontario, pour combattre cette menace aurait-il d'autre choix que de recourir aux méthodes de M. Duplessis avec leur teinte de fascisme ? ${ }^{15}$

13 Ibid., 3 février 1938.

14 Ibid., 11 avril 1938.

15 Ibid., 16 mars 1937. 
Avec la défaite du syndicalisme industriel en 1937 au cours de la grève de la société General Motors à Oshawa, la peur que McCullagh avait du communisme diminua quelque peu et il commença à interpréter la "loi de cadenas" et les agissements de Duplessis comme des menaces à la "national unity". Fin 1937, quand le premier ministre avertit qu'il ne se laisserait pas mener par les provinces de l'Ouest, il provoque une réaction presque hystérique du Globe and Mail: "Un fou politique obsédé par le pouvoir", Duplessis représente "la plus grave menace à la Confédération". ${ }^{16}$ McCullagh s'inquiétait de ce que les premiers ministres de l'Ontario et du Québec s'unissent contre Ottawa et les provinces moins riches. En donnant son appui à Mackenzie King et à la commission Rowell-Sirois, le Globe and Mail mettait en opposition l'attitude de dignité du Dominion et la hargne des deux provinces centrales. A partir de l'été de 1938, McCullagh voyait de la division partout. La "loi du cadenas" du Québec était "fasciste" et Aberhart et Hepburn avaient tous deux dépassé les bornes. Il supplia Hepburn et Duplessis de cesser de diviser le Canada et de se joindre dans un mouvement en faveur de la "national unity" et de "finances sûres". Retirant son appui antérieur au gouvernement fédéral, il disait que le gouvernement King n'avait pas désavoué la "loi du cadenas" par égard aux votes québécois qui lui étaient favorables. De plus, le gouvernement King, avec ses mesures d'assistance publique, avait détruit la confiance en soi et le caractère personnel. Le Globe and Mail conclut que la session parlementaire de 1939 devrait probablement représenter un pas vers le socialisme.

Convaincu que le Canada était déchiré par les forces destructrices du syndicalisme américain, des éléments étrangers subversifs et des leaders provinciaux bornés, McCullagh se tourna vers l'impérialisme. Car Berger a montré d'une manière fort convaincante, dans The Sense of Power, que l'impérialisme, au tournant du siècle, était une forme du nationalisme canadien. Cette affirmation semblerait particulièrement à propos pour ce qui est de l'impérialisme du Globe and Mail à la fin des années 1930. Car, bien que le Globe and Mail ait préconisé l'unité de l'empire et ait loué le pacifisme de la Grande-Bretagne, il n'a pas voulu que le Canada sacrifie sa souveraineté à l'Empire. Essentiellement son impérialisme représentait son inquiétude en face des problèmes du Canada. McCullagh défendait sa conception d'une société hiérarchique, anglo-saxonne, modérée, en opposition aux forces de changement issues de la dépression, en mettant l'accent sur le loyalisme, sur les vertus traditionnelles

16 Ibid., 17 décembre 1937. 
de la morale du travail, de la famille et de la vie rurale, sur le militarisme et la supériorité du fond de population britannique. Il était convaincu que les Canadiens comprendraient des mesures telles que la réduction du bilinguisme dans la fonction publique ou l'abolition des gouvernements provinciaux; il doutait fort qu'ils puissent se plaindre si on se servait du bon sens dans de telles matières. En préconisant l'usage de l'anglais, le Globe and Mail remarquait que l'extension de sa pratique courante favoriserait la diffusion de l'idéologie britannique. ${ }^{17}$ Le terme "unBritish" était particulièrement utile et pouvait être employé contre les Canadiens français, les syndicats américains ou quelque autre force subversive.

Les idées du Globe and Mail au sujet de l'immigration étaient fondées sur la crainte que le radicalisme soit largement répandu parmi les minorités canadiennes. Les colons britanniques devaient constituer une force imposante pour protéger le Canada contre la désunion et la subversion. Le Globe and Mail avait le sentiment que le Canada comptait trop de "nondescript travellers", de voyageurs non identifiés. D'accord avec le système australien où la préférence était donnée aux Britanniques, le Globe and Mail préconisa
a far-reaching program of economic development by which British genius would make a fresh start in the New World and establish another dominant Anglo-Saxon nation beside a great Anglo-Saxon neighbour. There is room for 60.000 .000 British people. ${ }^{18}$

Vers la fin des années 1930, le Globe and Mail était fatigué d'entendre parler des droits des minorités:
Canada must be regarded as a British-Canadian country in essence and principle... No one can deny the right and desirability of keeping alive memories of the homeland in song and folklore, but the laws, customs, and languages of the adopted country must become those of the new people if we are to build a united nation... Much is being said these days about the rights of the minority. It is time to speak of the rights of the majority. ${ }^{19}$

Bien que la majeure partie de ce qui précède ait été écrite pour répondre à des immigrants européens, aux syndicalistes américains ou aux partisans des minorités, le message s'adressait clairement aux Canadiens français; le Globe and Mail leur rap-

\footnotetext{
${ }^{17} \mathrm{Cf}$. volume 8 de Canadian Historical Readings: Brian J. Young,
}

"C. George McCullagh and the Leadership League".

18 Globe and Mail, 15 avril 1938.

19 Ibid., 20 juin 1937. 
pelait que c'était le régime britannique qui avait protégé leur nationalité. Partenaire dans la Confédération, le Québec avait eu la même chance que les autres provinces. Le séparatisme était tout bonnement une condamnation par les Canadiens français de leur incapacité à mettre en valeur les ressources sur lesquelles les ententes de la Confédération leur donnaient, comme majorité dans la province, un contrôle absolu..$^{20}$ N'ayant exprimé aucune doléance, ni en $1776 \mathrm{ni}$ en 1812, alors qu'il pouvait prendre les armes contre l'Angleterre, le Québec a une tradition de loyauté à maintenir.

Le Globe and Mail eut toujours beaucoup de mal à concilier le nationalisme des Canadiens français avec sa propre conception du nationalisme. On a interprété le premier comme une astuce de politiciens québécois qui menaçaient la "national unity". Encore une fois les "British values" fournissaient d'utiles slogans. Duplessis avait visité l'Angleterre et avait dû remarquer que le peuple britannique valait qu'on se batte pour lui; le Canada français aurait-il pu s'accroître de 70,000 à 3,000,000 d'âmes sous aucun autre régime étranger que le britannique ? Débordant de joie devant l'accueil chaleureux que le Québec avait réservé au Roi et à la Reine en mai 1939, le Globe and Mail décrivait l'événement comme un accueil britannique bien que canadienfrançais. ${ }^{21}$

Cette surabondance de clichés anglo-saxons et britanniques révélait chez McCullagh une réaction de défense. Il se voyait menacé dans sa conception de la société par le radicalisme de groupes minoritaires. L'attitude de son journal envers le Québec trouvait ses racines dans une mentalité d'assiégé plutôt que dans l'anticatholicisme traditionnellement associé avec le Globe et l'Ontario. Les éditorialistes de McCullagh comprenaient mal le Québec. Ils écrivaient davantage sur les mines de la province que sur son peuple. Ils brossaient rarement le portrait du Canadien français, et quand ils le faisaient, c'était pour louer son affabilité, sa gaieté et son style de vie fortement rural et familial. Si de temps à autre le Canada français faisait des déclarations alarmantes pour le milieu des affaires torontois, la raison en était que les Canadiens français étaient mal guidés par leurs leaders politiques intrigants. Quand il laissait entendre que les Canadiens français accepteraient de bon cœur l'abolition des gouvernements provinciaux, McCullagh semble avoir montré qu'il ignorait le véritable état des affaires plutôt qu'il ne manifestait une déception consciente.

${ }^{20}$ Ibid., 4 février 1937.

21 Ibid., 18 mai 1939. 
Carl Berger a refusé d'accepter l'idée d'un nationalisme économique qui aurait été la force motrice de l'impérialisme des années 1867-1914 et a expliqué les impérialistes de cette époque comme des hommes qui faisaient davantage cause commune avec les mouvements de protestation des agriculteurs qu'avec les grands capitalistes. Dans le cas de McCullagh, il est clair, tout au moins, que son conservatisme coïncida avec les intérêts économiques de ses compatriotes des milieux d'affaires torontois. Sa philosophie personnelle et ses rebâchages sur la morale du travail, de la famille et de la terre allaient de pair avec l'importance qu'il accordait aux valeurs britanniques. Il trouvait chez cellesci des armes utiles pour combattre le radicalisme. La "national unity", selon McCullagh, était synonyme d'intégration et d'assimilation de tous les groupes régionaux, ethniques et sociaux. La position du Globe and Mail vis-à-vis du Québec de 1935 à 1939 faisait partie d'une tentative, mêlée de nostalgie et d'agressivité, pour revenir aux années antérieures à 1930 . 